Optical resonances in microsphere photonic nanojets

This content has been downloaded from IOPscience. Please scroll down to see the full text. 2014 J. Opt. 16015704

(http://iopscience.iop.org/2040-8986/16/1/015704)

View the table of contents for this issue, or go to the journal homepage for more

Download details:

This content was downloaded by: qtsoon

IP Address: 130.88.106.243

This content was downloaded on 22/11/2013 at 12:08

Please note that terms and conditions apply. 


\title{
Optical resonances in microsphere photonic nanojets
}

\author{
Seoungjun Lee, Lin Li and Zengbo Wang \\ School of Mechanical, Aerospace and Civil Engineering, University of Manchester, \\ Manchester M13 9PL, UK \\ E-mail: seoungjun.lee@postgrad.manchester.ac.uk
}

Received 16 September 2013, accepted for publication 1 November 2013

Published 21 November 2013

\begin{abstract}
We report microsphere photonic nanojets, using the Mie theory to analyse the behaviour of the Poynting vector fields for incident visible wavelengths. Optical field structures of optical whirlpools and rhombuses are observed when incident plane waves transmit through $5 \mu \mathrm{m}$ and $30 \mu \mathrm{m}$ dielectric microspheres of various refractive indices from $n=1.4$ to 2.2 embedded in vacuum and water media. The waist of the photonic nanojets and the distance between the microsphere and the position of maximum electric intensity determines the potential for super-resolution imaging. These parameters vary with the refractive index and size of the dielectric microspheres. We have shown that the waists of the photonic nanojets are beyond the diffraction limit without significant optical diffraction.
\end{abstract}

Keywords: photonic nanojet, optical resonance, super-resolution, microsphere, diffraction limit

(Some figures may appear in colour only in the online journal)

\section{Introduction}

The spatial optical resolution of an imaging system is affected by the quality and configuration of the optics and more importantly by the diffraction limit. To resolve objects below the diffraction limit, metamaterial based super-lenses have been used to restore evanescent waves [1]. Microspheres have been successfully used for nano-fabrication [2, 3] and for super-resolution imaging [4]. Photonic nanojets can also approach sub-diffraction-limited detection enhanced by backscattering of light by dielectric micro-particles [5]. Backscattering enhancement induced by the photonic nanojets was experimentally demonstrated using barium titanate microspheres at visible wavelengths [6]. Dielectric microcylinders were also shown to be able to generate nano-scaled photonic jets and to enhance backscattering of light in the visible spectrum [7]. Such enhancements can overcome the optical diffraction limit without significant optical diffraction. The phenomenon of photonic nanojets was theoretically investigated in circular dielectric microcylinders [7, 8] and dielectric microspheres [9-12] with incident plane waves. The diffraction effect of a dielectric microsphere array was demonstrated in far-field photonic nanojets [13, 14]. Photonic nanojets were also experimentally confirmed with a polystyrene microsphere on a glass substrate with a confocal microscope [15]. The applications of photonic nanojets have been investigated in various fields, including optical data storage [16], two-photon fluorescence depletion microscopy [17], plasmonic nanodisc lithography [18], and optical forces at resonance and off-resonance wavelengths [19]. In this paper, we report the interactions of optical resonances and photonic nanojets that are generated by dielectric microspheres with diameters of $d=5$ and $30 \mu \mathrm{m}$ with refractive indices of $n_{1}=1.46$ (fused silica), 1.59 (polystyrene), $1.93\left(\mathrm{TiO}_{2}-\mathrm{BaO}-\mathrm{SiO}_{2}\right.$ glass), 2.20 $\left(\mathrm{TiO}_{2}-\mathrm{BaO}-\mathrm{ZnO}\right.$ glass) embedded in media with refractive indices of $n_{2}=1.0$ (vacuum) and 1.33 (water) for incident plane waves of wavelength $\lambda=300 \mathrm{~nm}, 400 \mathrm{~nm}, 500 \mathrm{~nm}$, $600 \mathrm{~nm}, 700 \mathrm{~nm}$ and $800 \mathrm{~nm}$, respectively. The distance between the microspheres and the position of maximum electric intensity (DMI) and full width at half maximum (FWHM) were determined. The potential for super-resolution imaging is explained by the analysis of the waist of the photonic nanojets and the optical field structures in the 
Poynting vectors in the near and near-external fields of a microsphere.

\section{Methods}

Light scattering and the optical cross-section of light interacting with a microsphere is modelled using the Mie theory by considering the electric $(\mathbf{E})$, magnetic $(\mathbf{H})$ and Poynting vector $(\mathbf{S}=\mathbf{E} \times \mathbf{H})$ fields. Using analytical Mie theory with Poynting vectors in the electromagnetic field, we show that high index dielectric microspheres with diameters of not only $5 \mu \mathrm{m}$ but also $30 \mu \mathrm{m}$ can achieve the photonic nanojet effect in the visible spectrum when illuminated with incident plane waves. Previously, photonic nanojets were limited to small particles below $10 \mu \mathrm{m}[6,7,10]$ because they could take advantage of producing a minimized FWHM and enhanced backscattering. The Poynting vector flow was used to analyse the field distribution from the direction of propagation [20, 21], because the image distribution of the Poynting vector can allow one to understand the local changes of the electromagnetic energy [22]. Thus, the capability for super-resolution imaging might be predicted with photonic nanojets in the near and near-external fields of a microsphere. In the simulations, when the incident plane waves propagate through a single dielectric microsphere along the $z$ coordinate, the flux of electric and magnetic vectors is along the $x$ and $y$ coordinates, respectively. The time-averaged Poynting vector is applied to the power per unit area by a wave. The single microsphere embedded in a medium is considered in the two-dimensional $x z$ plane. For the $30 \mu \mathrm{m}$ diameter microsphere, the boundary dimensions equal a square that is $72 \mu \mathrm{m}$ on each side, and the number of terms on each side is 2500 points. For the $5 \mu \mathrm{m}$ microsphere, the boundary dimensions are $12 \mu \mathrm{m}$, and the number of terms is 1000 points. The directional energy flux density is generated by streamlines and directional vector arrows. The distribution of the Poynting vectors was obtained, showing drastic transformations in the field distribution. This relationship includes several different parameters for the incident plane wavelengths, and the sizes and refractive indices of microspheres in different media, showing the optical resonances and complicated optical field structures of the near-field transformations [23, 24].

\section{Results and discussion}

The behaviour of the Poynting vectors in photonic nanojets for dielectric microspheres of diameter $d=5 \mu \mathrm{m}$ is shown in figure 1. The red coloured lines indicate high electric intensity and the blue coloured lines indicate low electric intensity. Dielectric microspheres with two different refractive indices of $n_{1}=1.46$ and $n_{1}=2.20$ are embedded in media with $n_{2}=1.0$ (vacuum) and $n_{2}=1.33$ (water) with incident plane waves of wavelength $\lambda_{2}=400 \mathrm{~nm}$. The optical field structures of near-field transformations that contain optical whirlpools are observed in the area of low electric intensity in the near and near-external field of the microsphere. The near-field transformations can be generated by interference between the evanescent and the propagating waves [20]. In figure 1(a), the dielectric microsphere with $d=5 \mu \mathrm{m}$ and refractive index $n_{1}=1.46$ in vacuum $\left(n_{2}=1.0\right)$ can clearly generate the transformation of optical whirlpools without significant diffraction in the near and near-external fields of the microsphere. Furthermore, it was experimentally confirmed to have super-resolution imaging through microsphere optical nanoscopy [4]. The interactions between the evanescent and the propagating waves can encourage photonic nanojets. This interference may provide a theoretical explanation of super-resolution imaging by transforming the evanescent waves from a near-field to a far-field propagating wave. Moreover, the resonance effect can be improved in the near field of the microsphere by this interference relationship [25]. In figure 1(b) with water $\left(n_{2}=\right.$ 1.33), the focal position is located far from the microsphere and optical whirlpools are not generated in the near field of the microsphere. The microsphere of $n_{1}=2.20$ refractive index in vacuum $\left(n_{2}=1.0\right)$ has the photonic nanojet focal point inside the microsphere, as shown in figure 1(c). Optical interference around the microsphere is generated so that a second focal position is observed near the top boundary of the microsphere. The second focal position can divert the energy of optical resonances so that the transformations of optical whirlpools may not obviously be generated. Moreover, the resonances surrounding a microsphere can decrease the backscattering enhancement of the photonic nanojets [6]. Thus, super-resolution imaging might be interrupted by the interference of the second focal position. In figure 1(d) with water $\left(n_{2}=1.33\right)$, the photonic nanojet is formed on the boundary of the microsphere. The optical whirlpools that are partially generated might not be sufficient for the near-field evanescent wave to propagate wave transformations.

The Poynting vector traces of photonic nanojets of dielectric microspheres of diameter $d=30 \mu \mathrm{m}$ are shown in figure 2. Optical whirlpools are clearly observed in figure 2(a) $n_{1}=1.59, n_{2}=1.0$ and (c) $n_{1}=2.20, n_{2}=1.33$. This could be the result of satisfactory interference between the evanescent and propagating waves, therefore providing super-resolution imaging to observe sub-diffraction-limit objects. In figure 2(b) with $n_{1}=1.59$ and $n_{2}=1.33$, the focal position is far from the microsphere and the optical whirlpools are observed in the near and near-external fields of the microsphere. This may cause insufficient near-field transformations. In figure 2(c) with $n_{1}=2.20$ and $n_{2}=$ 1.0 , the vector flow near the top of the microsphere is disturbed, similar to the phenomenon of figure 1(c). This may cause a change in the location of the focal position inside the microsphere. Optical whirlpool and rhombus structures are generated in the field of Poynting vectors around a microsphere. Optical whirlpool structures can be found at the bottom part inside the microsphere. Two optical whirlpool structures are observed each side of the light axis inside the microsphere with diameter $d=30 \mu \mathrm{m}$ in figure 2(a) $n_{1}=1.59, n_{2}=1.0$ and (d) $n_{1}=2.20$, $n_{2}=1.33$. Optical rhombus structures are created near the microsphere boundary. The location of the optical rhombuses depends on the refractive indices of the microsphere and 

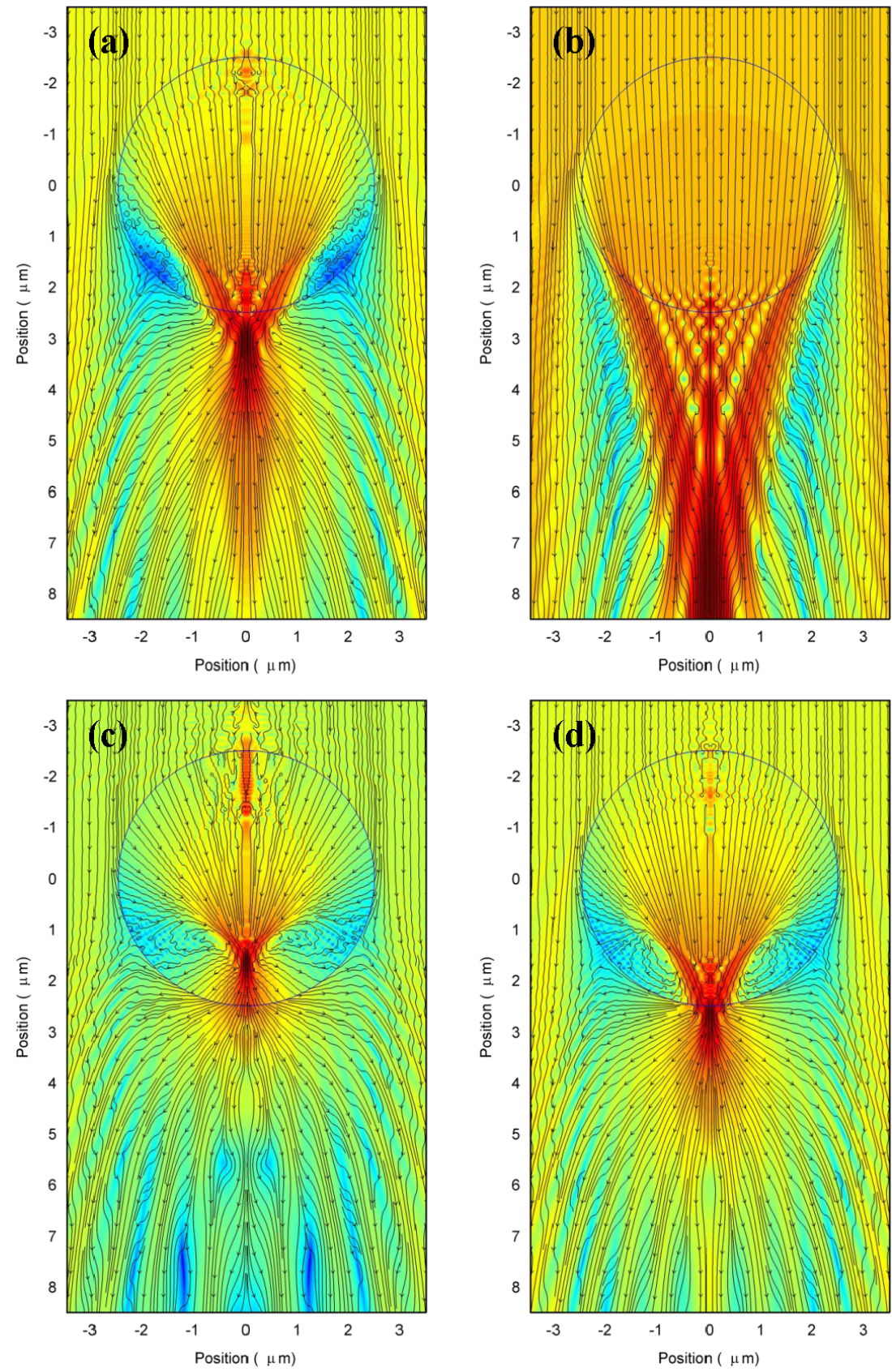

Figure 1. Poynting vectors of light transmitted through transparent dielectric microspheres of diameter $d=5 \mu \mathrm{m}$ and refractive index $n_{1}$ immersed in a medium of refractive index $n_{2}$. Incident plane waves of wavelength $\lambda_{2}=400 \mathrm{~nm}$ from the top. (a) $n_{1}=1.46, n_{2}=1.0$; (b) $n_{1}=1.46, n_{2}=1.33$; (c) $n_{1}=2.20, n_{2}=1.0 ;$ (d) $n_{1}=2.20, n_{2}=1.33$.

the medium. Optical rhombuses are also observed outside the microspheres in figure 2(b) $n_{1}=1.59, n_{2}=1.33$, and on the boundary in figure 2(a) $n_{1}=1.59, n_{2}=1.0$, and both inside and outside in figure 2(d) $n_{1}=2.20, n_{2}=$ 1.33. Such optical field structures can be clearly observed in larger diameter microspheres because of the high energy field intensity. When the sub-diffraction-limited photonic nanojet is generated in the microspheres, the optical whirlpool and rhombus structures appear together with vector streamlines. Such optical field structures can be generated by clear optical interactions through the microsphere that prevent the second focal positions and optical interference around the microsphere. Thus, optical rhombus and optical whirlpool structures may enhance optical interactions and contribute to the focusing of incident light to the microsphere. Furthermore, the energy flow can be disturbed by a second focal position if the focal position is inside the microsphere in figure 2(c) $n_{1}=2.20, n_{2}=1.0$. Such an interruption may break the optical field structures.

The FWHM and DMI of dielectric microspheres of diameter $d=5 \mu \mathrm{m}$ were determined with incident plane waves of wavelength $\lambda=300,400,500,600,700$ and $800 \mathrm{~nm}$, as shown in figure 3 . The interactions with microspheres with refractive indices of $n_{1}=1.46,1.59,1.93$, and 2.20 are 

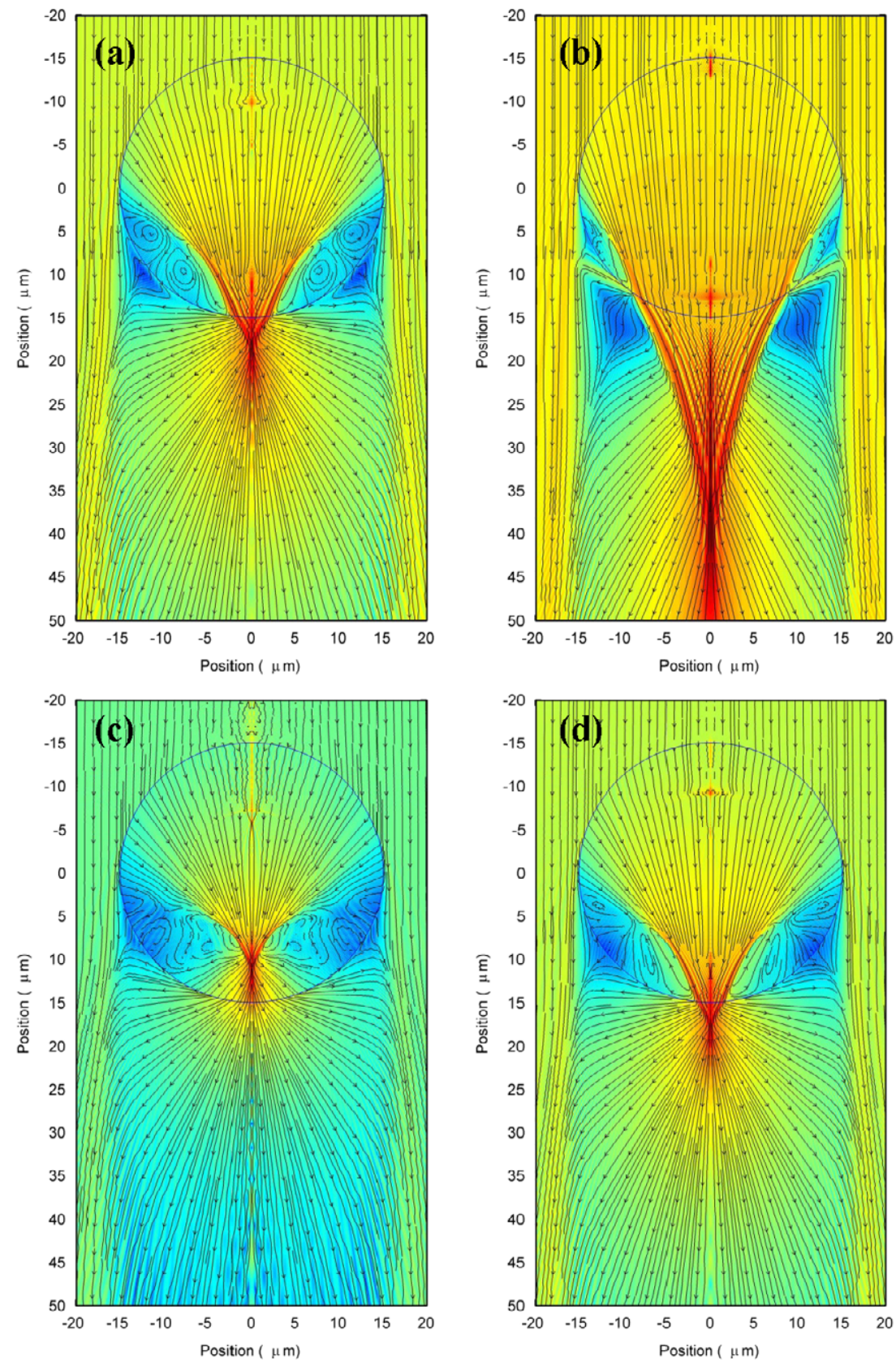

Figure 2. Poynting vector traces for plane wave light transmission through dielectric microspheres of diameter $d=30 \mu \mathrm{m}$ at a wavelength of $\lambda_{2}=400 \mathrm{~nm}$. (a) $n_{1}=1.59, n_{2}=1.0$; (b) $n_{1}=1.59, n_{2}=1.33$; (c) $n_{1}=2.20, n_{2}=1.0$; (d) $n_{1}=2.20, n_{2}=1.33$.

compared for media with refractive indices $n_{2}=1.0$ (vacuum) and $n_{2}=1.33$ (water). The FWHM is increased when the light wavelength is increased. On the other hand, the DMI can be slightly decreased when the wavelength is increased. This effect can be observed clearly for water $\left(n_{2}=1.33\right)$ because of optical aberrations. In vacuum $\left(n_{2}=1.0\right)$, the focal positions of $n_{1}=1.93$ and 2.20 are located inside the microsphere. It can be expected that near-field transformations may not occur between evanescent and propagating waves in the near and near-external fields of the microsphere, therefore it may not support super-resolution imaging.

For the dielectric microspheres with diameter $d=30 \mu \mathrm{m}$, the FWHM and DMI have been computed with incident plane waves in vacuum $\left(n_{2}=1.0\right)$ and water $\left(n_{2}=1.33\right)$, as shown in figure 4. The FWHM and DMI is increased as compared with the microspheres of diameter $d=5 \mu \mathrm{m}$. By increasing the size of the microspheres, the DMI can obviously emerge from the microspheres with refractive indices of $n_{1}=1.93$ and 2.20 and the activity of optical resonance can be stronger in the near and near-external field of the microspheres. As a result, clear optical whirlpools can be observed at the near horizontal plane of the microsphere.

The FWHMs divided by the incident wavelengths $(\lambda)$ for dielectric microspheres of diameters $d=5$ and $30 \mu \mathrm{m}$ are shown in table 1 . The refractive indices of microspheres and media are compared for wavelengths between 300 and 
(a)

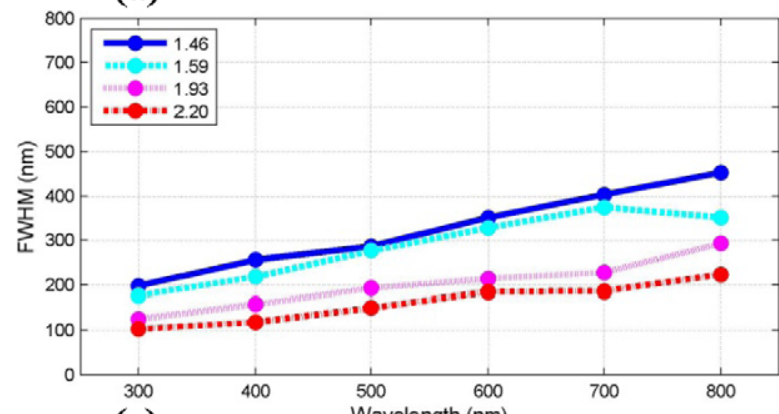

(c)

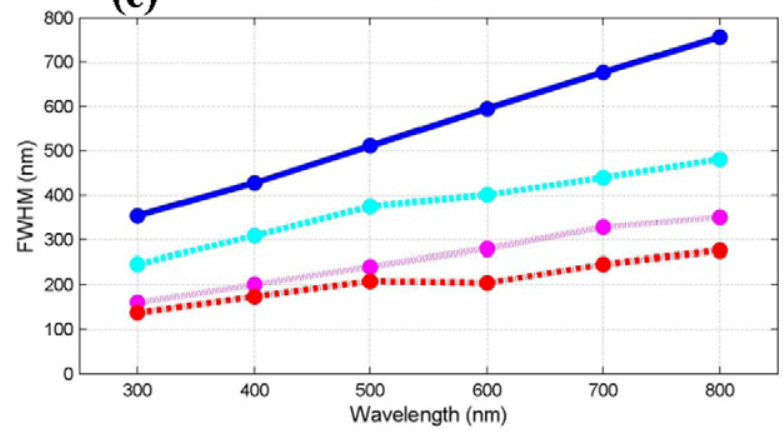

(b)

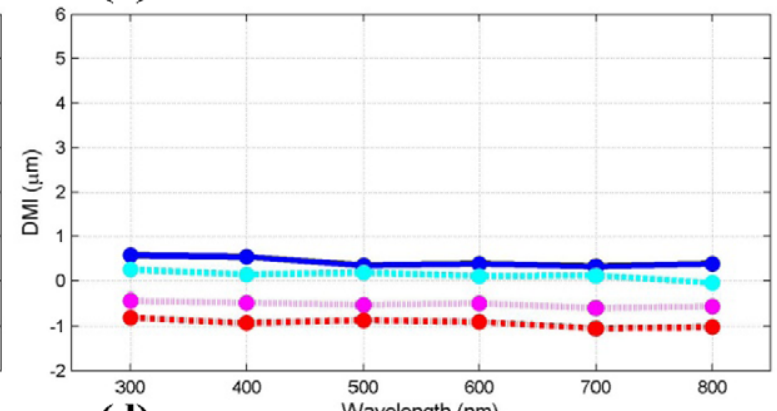

(d)

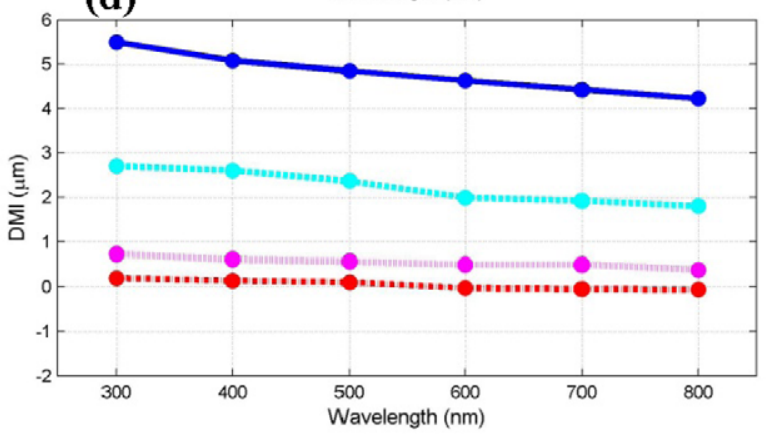

Figure 3. FWHMs and DMIs for dielectric spheres with a diameter $d=5 \mu \mathrm{m}$ with refractive indices of $n_{1}=1.46,1.59,1.93$, and 2.20, respectively, in vacuum $\left(n_{2}=1.0\right)$ and water $\left(n_{2}=1.33\right)$ at wavelengths $(\lambda)$ between 300 and $800 \mathrm{~nm}$. (a) and (b) $n_{2}=1.0 ;$ (c) and (d) $n_{2}=1.33$.

(a)

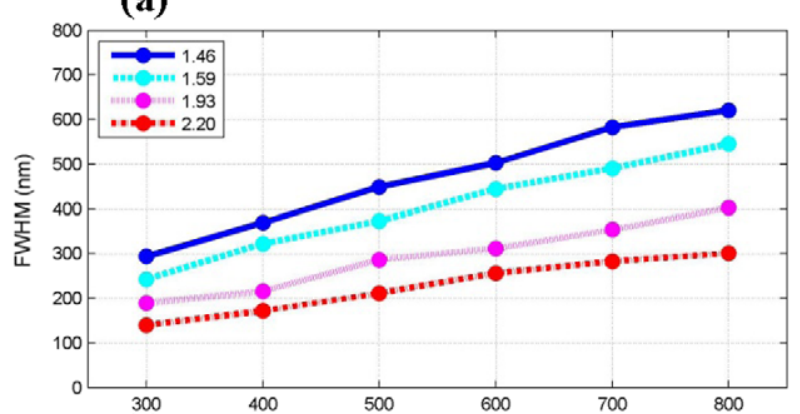

(c)

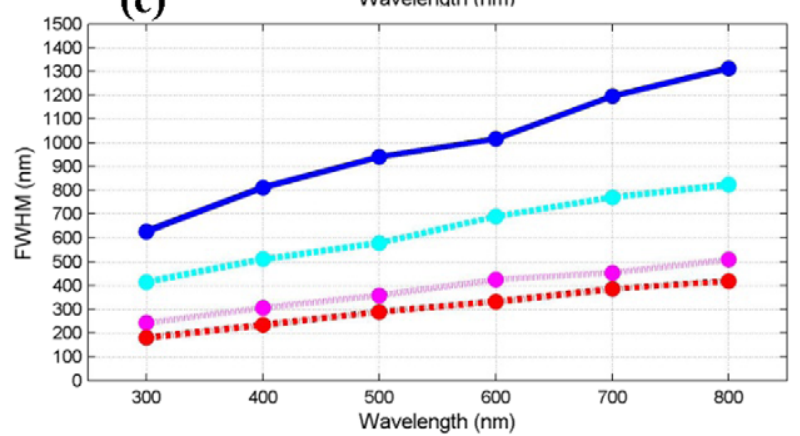

(b)

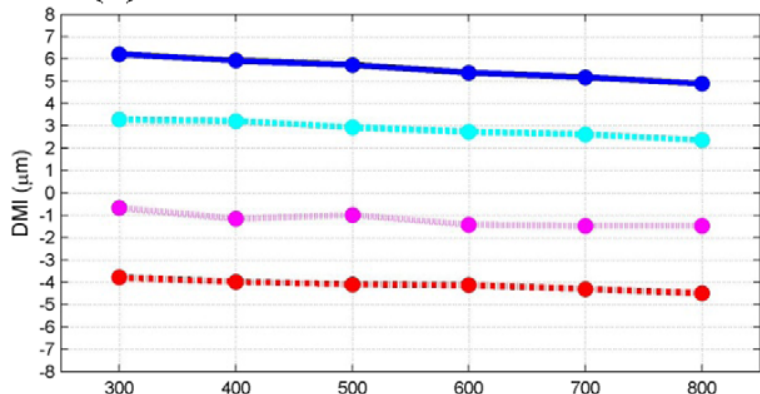

(d)

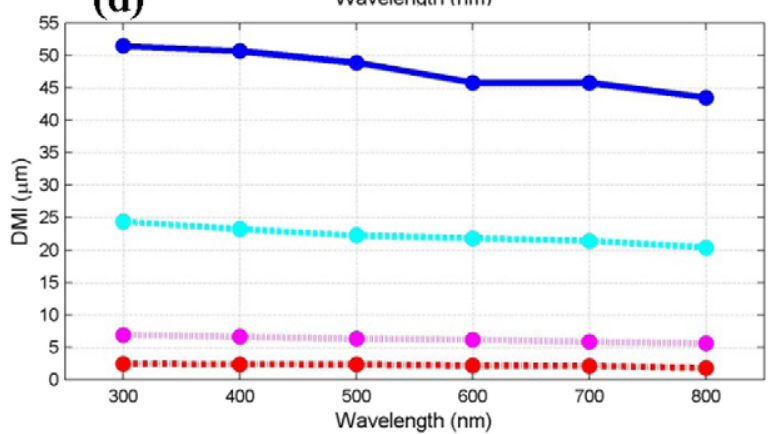

Figure 4. FWHMs and DMIs of dielectric microspheres with a diameter $d=30 \mu \mathrm{m}$ with four different refractive indices embedded in vacuum $\left(n_{2}=1.0\right)$ and water $\left(n_{2}=1.33\right)$ at wavelengths $300-800 \mathrm{~nm}$. (a) and (b) $n_{2}=1.0 ;$ (c) and (d) $n_{2}=1.33$.

$800 \mathrm{~nm}$. The value $\lambda_{2-5}$ in the visible spectrum is calculated from the average FWHM of $\lambda_{2}=400$ to $\lambda_{5}=700 \mathrm{~nm}$. The FWHM divided by the incident wavelength $(\lambda)$ seems to be independent of the wavelength, as the values are quite similar for wavelengths between 300 and $800 \mathrm{~nm}$. The values can be generally affected by the refractive indices of the microsphere and the media. The waist of the photonic nanojet is determined by the waist of the FWHM intensity [5]. In dielectric microspheres of diameter $d=5 \mu \mathrm{m}$, the waist of the photonic nanojet is similar to the diffraction limit for $n_{1}=1.46$ and 
Table 1. FWHM divided by the incident wavelength $(\lambda)$ for dielectric microspheres of diameter $d=5$ and $30 \mu \mathrm{m}$ in vacuum $\left(n_{2}=1.0\right)$ and water $\left(n_{2}=1.33\right.$ ). (a) $d=5 \mu \mathrm{m}, n_{2}=1.0$; (b) $d=5 \mu \mathrm{m}, n_{2}=1.33$; (c) $d=30 \mu \mathrm{m}, n_{2}=1.0$; (d) $d=30 \mu \mathrm{m}, n_{2}=1.33$.

\begin{tabular}{|c|c|c|c|c|c|c|c|}
\hline$n_{1}$ & $\begin{array}{l}\lambda_{1}= \\
300 \mathrm{~nm}\end{array}$ & $\begin{array}{l}\lambda_{2}= \\
400 \mathrm{~nm}\end{array}$ & $\begin{array}{l}\lambda_{3}= \\
500 \mathrm{~nm}\end{array}$ & $\begin{array}{l}\lambda_{4}= \\
600 \mathrm{~nm}\end{array}$ & $\begin{array}{l}\lambda_{5}= \\
700 \mathrm{~nm}\end{array}$ & $\begin{array}{l}\lambda_{6}= \\
800 \mathrm{~nm}\end{array}$ & $\begin{array}{l}\lambda_{2-5}= \\
\text { visible }\end{array}$ \\
\hline \multicolumn{8}{|c|}{ (a) $d=5 \mu \mathrm{m}, n_{2}=1.0$} \\
\hline 1.46 & $0.66 \lambda_{1}$ & $0.64 \lambda_{2}$ & $0.57 \lambda_{3}$ & $0.59 \lambda_{4}$ & $0.58 \lambda_{5}$ & $0.57 \lambda_{6}$ & $0.59 \lambda_{2-5}$ \\
\hline 1.59 & $0.59 \lambda_{1}$ & $0.55 \lambda_{2}$ & $0.55 \lambda_{3}$ & $0.55 \lambda_{4}$ & $0.53 \lambda_{5}$ & $0.44 \lambda_{6}$ & $0.55 \lambda_{2-5}$ \\
\hline 1.93 & $0.42 \lambda_{1}$ & $0.40 \lambda_{2}$ & $0.39 \lambda_{3}$ & $0.36 \lambda_{4}$ & $0.32 \lambda_{5}$ & $0.37 \lambda_{6}$ & $0.37 \lambda_{2-5}$ \\
\hline 2.20 & $0.34 \lambda_{1}$ & $0.30 \lambda_{2}$ & $0.30 \lambda_{3}$ & $0.31 \lambda_{4}$ & $0.27 \lambda_{5}$ & $0.28 \lambda_{6}$ & $0.29 \lambda_{2-5}$ \\
\hline \multicolumn{8}{|c|}{ (b) $d=5 \mu \mathrm{m}, n_{2}=1.33$} \\
\hline 1.46 & $1.18 \lambda_{1}$ & $1.07 \lambda_{2}$ & $1.02 \lambda_{3}$ & $0.99 \lambda_{4}$ & $0.97 \lambda_{5}$ & $0.95 \lambda_{6}$ & $1.01 \lambda_{2-5}$ \\
\hline 1.59 & $0.82 \lambda_{1}$ & $0.77 \lambda_{2}$ & $0.75 \lambda_{3}$ & $0.67 \lambda_{4}$ & $0.63 \lambda_{5}$ & $0.60 \lambda_{6}$ & $0.70 \lambda_{2-5}$ \\
\hline 1.93 & $0.54 \lambda_{1}$ & $0.50 \lambda_{2}$ & $0.48 \lambda_{3}$ & $0.47 \lambda_{4}$ & $0.47 \lambda_{5}$ & $0.44 \lambda_{6}$ & $0.48 \lambda_{2-5}$ \\
\hline 2.20 & $0.46 \lambda_{1}$ & $0.43 \lambda_{2}$ & $0.42 \lambda_{3}$ & $0.34 \lambda_{4}$ & $0.35 \lambda_{5}$ & $0.35 \lambda_{6}$ & $0.39 \lambda_{2-5}$ \\
\hline \multicolumn{8}{|c|}{ (c) $d=30 \mu \mathrm{m}, n_{2}=1.0$} \\
\hline 1.46 & $0.98 \lambda_{1}$ & $0.92 \lambda_{2}$ & $0.90 \lambda_{3}$ & $0.84 \lambda_{4}$ & $0.83 \lambda_{5}$ & $0.78 \lambda_{6}$ & $0.87 \lambda_{2-5}$ \\
\hline 1.59 & $0.81 \lambda_{1}$ & $0.81 \lambda_{2}$ & $0.75 \lambda_{3}$ & $0.74 \lambda_{4}$ & $0.70 \lambda_{5}$ & $0.68 \lambda_{6}$ & $0.75 \lambda_{2-5}$ \\
\hline 1.93 & $0.63 \lambda_{1}$ & $0.54 \lambda_{2}$ & $0.57 \lambda_{3}$ & $0.52 \lambda_{4}$ & $0.51 \lambda_{5}$ & $0.50 \lambda_{6}$ & $0.53 \lambda_{2-5}$ \\
\hline 2.20 & $0.47 \lambda_{1}$ & $0.43 \lambda_{2}$ & $0.42 \lambda_{3}$ & $0.43 \lambda_{4}$ & $0.40 \lambda_{5}$ & $0.38 \lambda_{6}$ & $0.42 \lambda_{2-5}$ \\
\hline \multicolumn{8}{|c|}{ (d) $d=30 \mu \mathrm{m}, n_{2}=1.33$} \\
\hline 1.46 & $2.09 \lambda_{1}$ & $2.03 \lambda_{2}$ & $1.88 \lambda_{3}$ & $1.69 \lambda_{4}$ & $1.71 \lambda_{5}$ & $1.64 \lambda_{6}$ & $1.83 \lambda_{2-5}$ \\
\hline 1.59 & $1.38 \lambda_{1}$ & $1.28 \lambda_{2}$ & $1.16 \lambda_{3}$ & $1.15 \lambda_{4}$ & $1.10 \lambda_{5}$ & $1.03 \lambda_{6}$ & $1.17 \lambda_{2-5}$ \\
\hline 1.93 & $0.81 \lambda_{1}$ & $0.77 \lambda_{2}$ & $0.72 \lambda_{3}$ & $0.71 \lambda_{4}$ & $0.65 \lambda_{5}$ & $0.64 \lambda_{6}$ & $0.71 \lambda_{2-5}$ \\
\hline 2.20 & $0.60 \lambda_{1}$ & $0.58 \lambda_{2}$ & $0.58 \lambda_{3}$ & $0.55 \lambda_{4}$ & $0.55 \lambda_{5}$ & $0.52 \lambda_{6}$ & $0.57 \lambda_{2-5}$ \\
\hline
\end{tabular}

1.59 in vacuum $\left(n_{2}=1.0\right)$. In dielectric microspheres of diameter $d=30 \mu \mathrm{m}$, the photonic nanojet can have a similar size to the diffraction limit for $n_{1}=2.20$ in water $\left(n_{2}=\right.$ 1.33). These photonic nanojets may have the capability for super-resolution imaging due to enhanced backscattering of visible light by sub-diffraction-limit objects [7].

\section{Conclusions}

We have demonstrated photonic nanojets and Poynting vector interactions for dielectric microspheres embedded in vacuum and water illuminated by plane waves with wavelengths ranging from the near-ultraviolet to the near-infrared. The photonic nanojet divided by the incident wavelength can be affected by the size and the refractive indices of the dielectric microspheres and the surrounding medium rather than by the incident plane wavelengths. Optical resonances including optical whirlpools and rhombuses are observed in Poynting vector fields by analytical Mie theory. For the dielectric microspheres of diameter $30 \mu \mathrm{m}$ with a refractive index of $n=$ 2.20 in water, and of diameter $5 \mu \mathrm{m}$ with refractive indices of $n=1.46$ and 1.59 in vacuum, the waist of the photonic nanojets is similar to the optical diffraction limit without significant optical diffraction and the second focal position on the microspheres. For dielectric microspheres with refractive indices of $n=2.20$ and 1.93 in vacuum, the waists of the photonic nanojets are smaller than the optical diffraction limit but the optical resonances can be disturbed because the second focal position can divert the energy of optical resonances. The phenomena of photonic nanojets from microspheres may provide potential applications for detecting sub-diffraction features with visible light in the fields of materials, biology, chemistry, and medical sciences.

\section{Appendix}

Following the formulae [26] used in the Mie theory, the calculation of the electromagnetic fields outside and inside a sphere is expressed with incident plane wave propagation along the $z$ coordinate, the electric vector $(\mathbf{E})$ field along the $x$ coordinate, and the magnetic vector $(\mathbf{H})$ field along the $y$ coordinate. The spherical coordinates $[r, \theta, \varphi]$ are indicated on the incident plane wave with the sphere positions for the electric and magnetic field,

$$
\begin{aligned}
& E_{r}=\mathrm{e}^{\mathrm{i} k_{\mathrm{m}} r \cos \theta} \sin \theta \cos \varphi, \\
& H_{r}=\sqrt{\varepsilon_{\mathrm{m}}} \mathrm{e}^{\mathrm{i} k_{\mathrm{m}} r \cos \theta} \sin \theta \sin \varphi, \\
& E_{\theta}=\mathrm{e}^{\mathrm{i} k_{\mathrm{m}} r \cos \theta} \cos \theta \cos \varphi, \\
& H_{\theta}=\sqrt{\varepsilon_{\mathrm{m}}} \mathrm{e}^{\mathrm{i} k_{\mathrm{m}} r \cos \theta} \cos \theta \sin \varphi, \\
& E_{\varphi}=-\mathrm{e}^{\mathrm{i} k_{\mathrm{m}} r \cos \theta} \sin \varphi, \\
& H_{\varphi}=-\sqrt{\varepsilon_{\mathrm{m}}} \mathrm{e}^{\mathrm{i} k_{\mathrm{m}} r \cos \theta} \sin \varphi .
\end{aligned}
$$

The wavevectors for the embedding medium, sphere and vacuum are $k_{m}=2 \pi \sqrt{\varepsilon_{\mathrm{m}}} / \lambda, k_{\mathrm{p}}=2 \pi \sqrt{\varepsilon_{\mathrm{p}}} / \lambda$ and $k_{0}=$ $2 \pi / \lambda$ respectively, where $\lambda$ is the radiation wavelength. The dielectric permittivities of the embedding medium and the sphere are $\varepsilon_{\mathrm{m}}$ and $\varepsilon_{\mathrm{p}}$ respectively, and the magnetic permeability is $\mu$. A dielectric sphere with radius $a$ is identified by the complex refractive index $\sqrt{\varepsilon_{\mathrm{m}}}=n_{\mathrm{m}}+\mathrm{i} k_{\mathrm{m}}$ for the medium and $\sqrt{\varepsilon_{\mathrm{p}}}=n_{\mathrm{p}}+\mathrm{i} k_{\mathrm{p}}$ for the sphere. 
A non-magnetic sphere $\left(\mu_{\mathrm{p}}=1\right)$ in vacuum (superscript $s$ for the scattered wave) gives

$$
\begin{aligned}
E_{r}^{(s)} & =\frac{\cos \varphi}{\left(k_{\mathrm{m}} r\right)^{2}} \sum_{n=1}^{\infty} n(n+1){ }^{e} B_{n} \zeta_{n}\left(k_{\mathrm{m}} r\right) P_{n}^{(1)}(\cos \theta) \\
E_{\theta}^{(s)} & =\frac{\cos \varphi}{k_{\mathrm{m}} r} \sum_{n=1}^{\infty}\left[{ }^{e} B_{n} \zeta_{n}^{\prime}\left(k_{\mathrm{m}} r\right) P_{n}^{(1)^{\prime}}(\cos \theta) \sin \theta-\mathrm{i}^{m} B_{n} \zeta_{n}\left(k_{\mathrm{m}} r\right) \frac{P_{n}^{(1)}(\cos \theta)}{\sin \theta}\right] \\
E_{\varphi}^{(s)} & =\frac{\sin \varphi}{k_{\mathrm{m}} r} \sum_{n=1}^{\infty}\left[{ }^{e} B_{n} \zeta_{n}^{\prime}\left(k_{\mathrm{m}} r\right) \frac{P_{n}^{(1)}(\cos \theta)}{\sin \theta}-\mathrm{i}^{m} B_{n} \zeta_{n}\left(k_{\mathrm{m}} r\right) P_{n}^{(1)^{\prime}}(\cos \theta) \sin \theta\right] \\
H_{r}^{(s)} & =\frac{\sqrt{\varepsilon_{\mathrm{m}}} \sin \varphi}{\left(k_{\mathrm{m}} r\right)^{2}} \sum_{n=1}^{\infty} n(n+1)^{m} B_{n} \zeta_{n}\left(k_{\mathrm{m}} r\right) P_{n}^{(1)}(\cos \theta) \\
H_{\theta}^{(s)} & =\mathrm{i} \frac{\sin \varphi}{k_{0} r} \sum_{n=1}^{\infty}\left[{ }^{e} B_{n} \zeta_{n}\left(k_{\mathrm{m}} r\right) \frac{P_{n}^{(1)}(\cos \theta)}{\sin \theta}+\mathrm{i}^{m} B_{n} \zeta_{n}^{\prime}\left(k_{\mathrm{m}} r\right) P_{n}^{(1)^{\prime}}(\cos \theta) \sin \theta\right] \\
H_{\varphi}^{(s)} & =\mathrm{i} \frac{\cos \varphi}{k_{0} r} \sum_{n=1}^{\infty}\left[{ }^{e} B_{n} \zeta_{n}\left(k_{\mathrm{m}} r\right) P_{n}^{(1)^{\prime}}(\cos \theta) \sin \theta+\mathrm{i}^{m} B_{n} \zeta_{n}^{\prime}\left(k_{\mathrm{m}} r\right) \frac{P_{n}^{(1)}(\cos \theta)}{\sin \theta}\right]
\end{aligned}
$$

The inside of the non-magnetic sphere (superscript $p$ for the scattered wave) is given by

$$
\begin{aligned}
E_{r}^{(p)} & =\frac{\cos \varphi}{\left(k_{\mathrm{p}} r\right)^{2}} \sum_{n=1}^{\infty} n(n+1)^{e} A_{n} \Psi_{n}\left(k_{\mathrm{p}} r\right) P_{n}^{(1)}(\cos \theta) \\
E_{\theta}^{(p)} & =\frac{\cos \varphi}{k_{\mathrm{p}} r} \sum_{n=1}^{\infty}\left[{ }^{e} A_{n} \Psi_{n}^{\prime}\left(k_{\mathrm{p}} r\right) P_{n}^{(1)^{\prime}}(\cos \theta) \sin \theta-\mathrm{i}^{m} A_{n} \Psi_{n}\left(k_{\mathrm{p}} r\right) \frac{P_{n}^{(1)}(\cos \theta)}{\sin \theta}\right] \\
E_{\varphi}^{(p)} & =\frac{\sin \varphi}{k_{\mathrm{p}} r} \sum_{n=1}^{\infty}\left[{ }^{e} A_{n} \Psi_{n}^{\prime}\left(k_{\mathrm{p}} r\right) \frac{P_{n}^{(1)}(\cos \theta)}{\sin \theta}-\mathrm{i}^{m} A_{n} \Psi_{n}\left(k_{p} r\right) P_{n}^{(1)^{\prime}}(\cos \theta) \sin \theta\right] \\
H_{r}^{(p)} & =\frac{\sqrt{\varepsilon_{\mathrm{m}}} \sin \varphi}{\left(k_{\mathrm{p}} r\right)^{2}} \sum_{n=1}^{\infty} n(n+1){ }^{m} A_{n} \Psi_{n}\left(k_{\mathrm{p}} r\right) P_{n}^{(1)}(\cos \theta), \\
H_{\theta}^{(p)} & =\mathrm{i} \frac{\sin \varphi}{k_{0} r} \sum_{n=1}^{\infty}\left[{ }^{e} A_{n} \Psi_{n}\left(k_{\mathrm{p}} r\right) \frac{P_{n}^{(1)}(\cos \theta)}{\sin \theta}+\mathrm{i}^{m} A_{n} \Psi_{n}^{\prime}\left(k_{\mathrm{p}} r\right) P_{n}^{(1)}(\cos \theta) \sin \theta\right] \\
H_{\varphi}^{(p)} & =\mathrm{i} \frac{\cos \varphi}{k_{0} r} \sum_{n=1}^{\infty}\left[{ }^{e} A_{n} \Psi_{n}\left(k_{\mathrm{p}} r\right) P_{n}^{(1)^{\prime}}(\cos \theta) \sin \theta+\mathrm{i}^{m} A_{n} \Psi_{n}^{\prime}\left(k_{\mathrm{p}} r\right) \frac{P_{n}^{(1)}(\cos \theta)}{\sin \theta}\right]
\end{aligned}
$$

with the coefficients

$$
\begin{aligned}
{ }^{e} B_{n} & =\mathrm{i}^{n+1} \frac{2 n+1}{n(n+1)} a_{n}, & { }^{m} B_{n} & =\mathrm{i}^{n+1} \frac{2 n+1}{n(n+1)} b_{n}, \\
{ }^{e} A_{n} & =\mathrm{i}^{n+1} \frac{2 n+1}{n(n+1)} c_{n}, & { }^{m} A_{n} & =\mathrm{i}^{n+1} \frac{2 n+1}{n(n+1)} d_{n},
\end{aligned}
$$

where

$$
\begin{aligned}
a_{n} & =\frac{y \Psi_{n}^{\prime}(x) \Psi_{n}(y)-x \Psi_{n}(x) \Psi_{n}^{\prime}(y)}{y \zeta_{n}^{\prime}(x) \Psi_{n}(y)-x \Psi_{n}^{\prime}(y) \zeta_{n}(x)}, \\
b_{n} & =\frac{y \Psi_{n}^{\prime}(y) \Psi_{n}(x)-x \Psi_{n}(y) \Psi_{n}^{\prime}(x)}{y \Psi_{n}^{\prime}(y) \zeta_{n}(x)-x \Psi_{n}(y) \zeta_{n}^{\prime}(x)}, \\
c_{n} & =\frac{y \zeta_{n}(x) \Psi_{n}^{\prime}(x)-y \zeta_{n}^{\prime}(x) \Psi_{n}(x)}{y \zeta_{n}^{\prime}(x) \Psi_{n}(y)-x \Psi_{n}^{\prime}(y) \zeta_{n}(x)}, \\
d_{n} & =\frac{y \zeta_{n}^{\prime}(x) \Psi_{n}(x)-y \zeta_{n}(x) \Psi_{n}^{\prime}(x)}{y \Psi_{n}^{\prime}(y) \zeta_{n}(x)-x \Psi_{n}(y) \zeta_{n}^{\prime}(x)}, \quad \zeta_{n}^{\prime}(\rho)=\frac{\partial \zeta_{n}(\rho)}{\partial \rho} \\
x & =k_{\mathrm{m}} a, \quad{k_{\mathrm{p}}} a, \\
\zeta_{n}(\rho) & =\rho h_{n}^{(1)}(\rho)=\sqrt{\frac{\pi \rho}{2}} H_{n+\frac{1}{2}}^{(1)}(\rho), \quad \Psi_{n}^{\prime}(\rho)=\frac{\partial \Psi_{n}(\rho)}{\partial \rho}, \\
\Psi_{n}(\rho) & =\rho j_{n}(\rho)=\sqrt{\frac{\pi \rho}{2}} J_{n+\frac{1}{2}}(\rho), \quad
\end{aligned}
$$


are the scattering coefficients and we make use of

(i) the associated Legendre function,

$$
\begin{aligned}
P_{n}^{m}(\cos \theta) \equiv & \frac{\left(1-(\cos \theta)^{2}\right)^{\frac{m}{2}}}{2^{n} n !} \\
& \times \frac{d^{n+m}}{d(\cos \theta)^{n+m}}\left((\cos \theta)^{2}-1\right)^{n}, \\
& (m \geq 0) \\
P_{n}^{m}(\cos \theta) \equiv & (-1)^{-m} \frac{(n+m) !}{(n-m) !} P_{n}^{-m}(\cos \theta), \\
& (m<0)
\end{aligned}
$$

(ii) the spherical Hankel function,

$$
h_{n}^{(1)}(\rho)=\sqrt{\frac{\pi}{2 \rho}} H_{n+\frac{1}{2}}^{(1)}(\rho),
$$

(iii) the spherical Bessel function,

$$
j_{n}(\rho)=\sqrt{\frac{\pi}{2 \rho}} J_{n+\frac{1}{2}}(\rho) .
$$

\section{References}

[1] Pendry J B 2000 Negative refraction makes a perfect lens Phys. Rev. Lett. 85 3966-9

[2] Guo W et al 2008 Chemical-assisted laser parallel nanostructuring of silicon in optical near fields Nanotechnology 19455302

[3] Li L et al 2011 Laser nano-manufacturing — state of the art and challenges CIRP Ann.-Manuf. Technol. 60 735-55

[4] Wang Z et al 2011 Optical virtual imaging at $50 \mathrm{~nm}$ lateral resolution with a white-light nanoscope Nature Commun. 2218

[5] Heifetz A et al 2009 Photonic nanojets J. Comput. Theor. Nanosci. 6 1979-92

[6] Yang S, Taflove A and Backma V 2011 Experimental confirmation at visible light wavelengths of the backscattering enhancement phenomenon of the photonic nanojet Opt. Express 19 7084-93

[7] Chen Z, Taflove A and Backman V 2004 Photonic nanojet enhancement of backscattering of light by nanoparticles: a potential novel visible-light ultramicroscopy technique Opt. Express 12 1214-20

[8] Itagi A V and Challener W A 2005 Optics of photonic nanojets J. Opt. Soc. Am. A 22 2847-58
[9] Geints Y E, Panina E K and Zemlyanov A A 2010 Control over parameters of photonic nanojets of dielectric microspheres Opt. Commun. 283 4775-81

[10] Li X et al 2005 Optical analysis of nanoparticles via enhanced backscattering facilitated by $3 \mathrm{D}$ photonic nanojets $O p t$. Express 13 526-33

[11] Lecler S, Takakura Y and Meyrueis P 2005 Properties of a three-dimensional photonic jet Opt. Lett. 30 2641-3

[12] Kong S C, Taflove A and Backman V 2009 Quasi one-dimensional light beam generated by a graded-index microsphere Opt. Express 17 3722-31

[13] Yannopapas V 2012 Photonic nanojets as three-dimensional optical atom traps: a theoretical study Opt. Commun. $2852952-5$

[14] Wang T et al 2011 Subwavelength focusing by a microsphere array J. Opt. 13035702

[15] Ferrand P et al 2008 Direct imaging of photonic nanojets Opt. Express 16 6930-40

[16] Kong S C et al 2008 Photonic nanojet-enabled optical data storage Opt. Express 16 13713-9

[17] Kuang C et al 2012 Creating attoliter detection volume by microsphere photonic nanojet and fluorescence depletion Opt. Commun. 285 402-6

[18] Kim J et al 2012 Fabrication of plasmonic nanodiscs by photonic nanojet lithography App. Phys. Express 5025201

[19] Cui X, Erni D and Hafner C 2008 Optical forces on metallic nanoparticles induced by a photonic nanojet Opt. Express $1613560-8$

[20] Yang M C and Webb K J 2005 Poynting vector analysis of a superlens Opt. Lett. 30 2382-4

[21] Sztul H I and Alfano R R 2008 The Poynting vector and angular momentum of Airy beams Opt. Express 16 9411-6

[22] Seo M A et al 2007 Fourier-transform terahertz near-field imaging of one-dimensional slit arrays: mapping of electric-field-, magnetic-field-, and Poynting vectors $O p t$. Express 15 11781-9

[23] Luk'yanchuk B S and Ternovsky V 2006 Light scattering by a thin wire with a surface-plasmon resonance: bifurcations of the Poynting vector field Phys. Rev. B 73235432

[24] Bashcvoy M V, Fedotov V A and Zheludev N I 2005 Optical whirlpool on an absorbing metallic nanoparticle Opt. Express 13 8372-9

[25] Ben-Aryeh Y 2008 Transmission enhancement by conversion of evanescent waves into propagating waves Appl. Phys. B 91 157-65

[26] Luk'yanchuk B S, Zheng Y W and Lu Y 2000 Laser cleaning of solid surface: optical resonance and near-field effects Proc. SPIE 4065 576-87 